Case Report

\title{
Correlation of cariogenic bacteria and dental caries in adults
}

\author{
Fusao Nishikawara ${ }^{1,2)}$, Seiko Katsumura ${ }^{3)}$, Ayumi Ando ${ }^{4)}$, Yoh Tamaki ${ }^{1,2,5)}$, \\ Yoshiki Nakamura $^{6)}$, Keita Sato ${ }^{7)}$, Yoshiaki Nomura ${ }^{1,2)}$ and Nobuhiro Hanada ${ }^{2)}$ \\ ${ }^{1)}$ Department of Preventive Dentistry and Public Health, School of Dental Medicine, Tsurumi University, \\ Kanagawa, Japan \\ ${ }^{2)}$ Department of Oral Health, National Institute of Public Health, Saitama, Japan \\ ${ }^{3)}$ Department of Anatomy, School of Dental Medicine, Tsurumi University, Kanagawa, Japan \\ ${ }^{4)}$ Ando Dental Office, Iwate, Japan \\ ${ }^{5)}$ Division of Oral Health, Department of Health Science, Kanagawa Dental College, Kanagawa, Japan \\ ${ }^{6}$ ) Department of Orthodontics, School of Dental Medicine, Tsurumi University, Kanagawa, Japan \\ ${ }^{7)}$ Department of Forensic Medicine and Dentistry, School of Dental Medicine, \\ Tsurumi University, Kanagawa, Japan
}

(Received 30 March and accepted 11 September 2006)

\begin{abstract}
Many studies suggest that mutans streptococci (MS), Lactobacillus (LB), and salivary buffering capacity are important risk factors for dental caries. However, target populations for most studies were children. In adult patients, the same risk factors affect the number of fillings or prostheses or secondary caries. It is therefore important to investigate these risk factors as predictors of caries in adults. In the present study, we evaluated the oral conditions of adult subjects at private dental offices using bite-wing radiographs. Detection of salivary LB level using Dentocult LB had a statistically significant correlation with the number of flat surface caries and approximal caries $(P<\mathbf{0 . 0 0 1})$. Detection of salivary MS level using Dentocult MS and salivary buffering capacity did not predict dental caries. Thus, detection of salivary LB level using Dentocult LB may be a useful tool for detecting approximal and secondary caries. (J. Oral Sci. 48, 245251, 2006)
\end{abstract}

Keywords: adults; dental caries; mutans streptococci; Lactobacillus.

Correspondence to Dr. Fusao Nishikawara, Department of Preventive Dentistry and Public Health, School of Dental Medicine, Tsurumi University, 2-1-3 Tsurumi, Tsurumi-ku, Yokohama, Kanagawa 230-8501, Japan

Tel: +81-45-580-8379

Fax: +81-45-573-9599

E-mail: fnishy@yahoo.co.jp

\section{Introduction}

Epidemiological studies of risk factors for dental caries have focused mainly on salivary levels of cariogenic bacteria as predictors (1-3). It has been suggested that salivary levels of mutans streptococci (MS) and Lactobacillus (LB) correlate with the number of decayed teeth or the number of decayed, filled or missing teeth (DMF) (4). The group MS includes Streptococcus mutans ( $S$. mutans) and Streptococcus sobrinus (S. sobrinus) and their virulence factors are well known as they exist within an oral biofilm on the tooth surface. Key virulence factors are acidogenicity, acid tolerance and synthesis of water insoluble glucan from sucrose (5-7). Meanwhile, LB does not avidly colonize teeth surfaces, but they may be transiently found in the oral cavity even before teeth erupt. They preferentially colonize the dorsum of the tongue and are carried into saliva by the sloughing of the tongue epithelium (8). Their numbers in saliva appear to reflect the consumption of simple carbohydrates by the host $(9,10)$. LB is highly acidogenic in the presence of carbohydrates, they are acid tolerant (11), and they are often isolated from established carious lesions (12). Some LB strains are cariogenic in experimental animals. Their cariogenicity is dependent upon the consumption of carbohydrate rich diets by the animal (13). It is also known that large amounts of MS and LB inhabit caries lesions (14). However, a previous study reported that the detection of MS was site specific, while LB was rarely isolated, in 
early caries (15). Commercially available chair side kits, Dentocult SM and Dentocult LB, have often been used to estimate levels of these bacteria to evaluate their ability to serve as a predictor of dental caries $(16,17)$.

Based on the results of these kits, many studies have predicted the future incidence of dental caries (18-20). However, few studies have shown a correlation between the results of these kits and presence of dental caries (4).

Most study populations for prediction were children (21-23), while few studies included adults $(24,25)$. This could be due to the fact that the mechanisms of pathogenesis in adults were so much more complicated than children. In adults, secondary caries and root caries occurred more frequently than primary caries. Moreover, smoking and medication which affect the salivary flow, may increase the risk of dental caries $(26,27)$. Most studies evaluating oral levels of these bacteria were carried out as field studies. Under such conditions as mass check-ups, precise evaluation of dental caries is impossible. In children and adults, there was no difference in the proportion of LB in saliva (8). Therefore, adults may have a similar caries risk to that of children. Moreover, root caries in elderly people are due to their having prosthetic crowns, or non-use of interdental brushes and dental floss (19). Also, in adults, oral conditions are affected by restorations and prostheses. This may make it difficult to evaluate the incidence of new dental caries and dental caries already existing as secondary caries. In our study, dental caries were more precisely evaluated at dental offices with the aid of radiographs. The aim of this study was to evaluate the determination of salivary MS level using Dentocult MS, salivary LB level using Dentocult LB and buffering capacity as clinical indicators of the present dental caries condition, especially in adult patients with complicated oral conditions.

\section{Materials and Methods}

\section{Study population and oral examination}

The study population consisted of patients attending private dental offices for the treatment of caries. We enrolled 152 adult subjects ( 67 males and 85 females), who were generally healthy and had good oral conditions. The mean age was $36.1 \pm 12.6$ years old (age range: 20 to 63 years old). The subjects were informed about the aim of this study well in advance and signed consent forms. At the first visit, the number of decayed, missing and filled teeth (number of DMF teeth for adults) was recorded for each subject after oral examination using light and exploration using dental mirrors according to WHO criteria (28). Levels of dental plaque were evaluated by O'Leary's Plaque Control Record (PCR) with the aid of disclosing agents. Full mouth oral radiographs were taken using the bitewing method to precisely detect approximal caries $(29,30)$. In Japan, conventionally, dental caries are classified according to their progression. We used these criteria and assigned subjects into one of two groups; CA: those who showed demineralization of enamel surfaces and dentinal caries CB: those who showed dental caries extending into the pulp and tooth stumps. We classified dental caries into flat surface caries and approximal caries. The number of teeth involved and the number of surfaces analyzed were correlated with the oral levels of cariogenic bacteria.

\section{Evaluation of cariogenic bacteria and buffering capacity}

Stimulated whole saliva samples were obtained from subjects by chewing for $5 \mathrm{~min}$ on wax blocks contained in the commercial kits. Salivary levels of MS and LB were estimated by Dentocult SM and Dentocult LB (Orion Diagnostica Co. Ltd, Epsom, Finland), respectively. The time of incubation for Dentocult SM was 48 hours and that for Dentocult LB was 96 hours. Evaluation of MS and LB levels was done using the model chart in the instruction manual. Salivary buffering capacity was evaluated using Dentobuff strips (Orion Diagnostica Co. Ltd, Epsom, Finland).

\section{Statistical analysis}

Kruskal-Wallis tests were used to evaluate the correlation between oral conditions and levels of cariogenic bacteria or salivary buffering capacity. We classified dental caries into several groups: primary caries or secondary caries, flat surface caries or approximal caries. Logistic regression analysis was used to evaluate the odds ratios of these factors for dental caries. Multiple logistic regression analysis was carried out to simultaneously evaluate the correlation of each dental caries condition. All of these analyses were carried out using SPSS software Ver. 14.0 (SPSS Co. Ltd Tokyo, Japan).

\section{Results}

The correlation between salivary levels of MS, LB, salivary buffering capacity and the number of decayed teeth, missing teeth, or teeth with fillings (DMFT) was determined. As shown in Table 1, determination of salivary MS level using Dentocult MS was significantly correlated with PCR $(P=0.016)$. We observed a tendency for the DMFT score to increase with an increase in the salivary levels of MS. Salivary LB level determined using Dentocult LB showed statistically significant correlations with PCR $(P=0.007)$, DMFT $(P=0.020)$, number of decayed teeth $(P=0.018)$ and number of approximal caries $(P=0.005)$ (Table 2). Similar to MS, we observed a tendency for the 
DMFT score to increase with an increase in LB. Salivary buffering capacity had a significant correlation with the number of flat surface caries $(P=0.004)$ and approximal caries $(P=0.026)$ (Table 3$)$.

Table 1 Correlation of oral conditions and detection of salivary MS level using Dentocult MS

\begin{tabular}{|c|c|c|c|c|c|c|c|c|c|c|c|}
\hline \multirow{2}{*}{ MS } & \multicolumn{2}{|c|}{$0(n=31)$} & \multicolumn{2}{|c|}{$1(n=40)$} & \multicolumn{2}{|c|}{$2(n=56)$} & \multicolumn{2}{|c|}{$3(n=30)$} & \multicolumn{3}{|c|}{ Total $(n=157)$} \\
\hline & mean & SD & mean & SD & mean & SD & mean & SD & mean & SD & $P$-value \\
\hline Age at the first visit & 36.770 & 11.514 & 33.670 & 11.439 & 38.360 & 13.145 & 37.670 & 13.535 & 36.720 & 12.518 & 0.385 \\
\hline $\mathrm{D}$ & 3.700 & 4.268 & 3.900 & 3.078 & 4.050 & 3.009 & 4.900 & 3.913 & 4.110 & 3.450 & 0.381 \\
\hline M & 2.300 & 2.959 & 1.130 & 1.870 & 1.840 & 2.192 & 1.550 & 2.501 & 1.680 & 2.343 & 0.221 \\
\hline $\mathrm{F}$ & 8.520 & 5.381 & 10.600 & 6.054 & 11.180 & 5.809 & 11.760 & 5.540 & 10.660 & 5.795 & 0.069 \\
\hline DMFT & 14.520 & 6.606 & 15.620 & 7.228 & 17.000 & 6.697 & 18.210 & 7.078 & 16.420 & 6.943 & 0.129 \\
\hline Total of flat surface caries & 2.000 & 4.405 & 2.400 & 4.528 & 2.790 & 4.111 & 2.330 & 4.727 & 2.450 & 4.367 & 0.398 \\
\hline Total of approximal caries & 3.920 & 6.202 & 6.100 & 6.476 & 6.480 & 7.527 & 5.970 & 6.184 & 5.780 & 6.771 & 0.232 \\
\hline PCR & 32.160 & 18.664 & 34.410 & 14.700 & 42.280 & 20.838 & 44.560 & 21.578 & 38.710 & 19.623 & 0.016 \\
\hline
\end{tabular}

Scores were evaluated for salivary levels of MS using the chart of the Dentocult SM kit.

Score 0 or $1:<10^{5} \mathrm{CFU} / \mathrm{ml}$, Score 2: $10^{5}-10^{6} \mathrm{CFU} / \mathrm{ml}$, Score $3: 10^{6}<\mathrm{CFU} / \mathrm{ml}$

No statistically significant correlations were found for primary and secondary caries.

DMFT scores increased with increases in salivary MS level determined using Dentocult MS. Detection of salivary MS level using

Dentocult MS was significantly correlated with PCR record but not other variables.

Table 2 Correlation of oral conditions and detection of salivary LB level using Dentocult LB

\begin{tabular}{|c|c|c|c|c|c|c|c|c|c|c|c|}
\hline \multirow{2}{*}{ LB } & \multicolumn{2}{|c|}{$0(n=73)$} & \multicolumn{2}{|c|}{$1(n=32)$} & \multicolumn{2}{|c|}{$2(n=40)$} & \multicolumn{2}{|c|}{$3(n=12)$} & \multicolumn{3}{|c|}{ Total $(n=157)$} \\
\hline & mean & SD & mean & SD & mean & SD & mean & SD & mean & SD & $P$-value \\
\hline Age at the first visit & 35.790 & 11.976 & 38.590 & 14.036 & 36.980 & 13.077 & 36.500 & 10.282 & 36.720 & 12.518 & 0.882 \\
\hline $\mathrm{M}$ & 1.570 & 2.097 & 1.550 & 1.997 & 2.050 & 2.964 & 1.420 & 2.392 & 1.680 & 2.343 & 0.929 \\
\hline $\mathrm{F}$ & 10.320 & 5.669 & 10.000 & 4.796 & 11.180 & 6.117 & 12.670 & 7.750 & 10.660 & 5.795 & 0.481 \\
\hline Total of flat surface caries & 1.120 & 2.273 & 2.190 & 3.042 & 2.720 & 3.651 & 10.250 & 9.206 & 2.450 & 4.367 & 0.000 \\
\hline Total of approximal caries & 4.510 & 5.513 & 5.000 & 5.105 & 5.950 & 6.683 & 15.080 & 10.604 & 5.780 & 6.771 & 0.005 \\
\hline PCR & 33.300 & 18.674 & 42.520 & 24.204 & 43.080 & 16.866 & 46.880 & 11.821 & 38.710 & 19.623 & 0.007 \\
\hline
\end{tabular}

Scores were evaluated for salivary levels of LB using the chart of the Dentocult LB kit.

Score 0: $10^{3} \mathrm{CFU} / \mathrm{ml}$, Score 1: $10^{4} \mathrm{CFU} / \mathrm{ml}$, Score 2: $10^{5} \mathrm{CFU} / \mathrm{ml}$, Score $3: 10^{6}<\mathrm{CFU} / \mathrm{ml}$

There were no statistically significant correlations between LB levels and primary and secondary caries.

DMFT scores increased with increases in detection of salivary LB level using Dentocult LB. Determination of salivary LB level using

Dentocult LB showed significant correlations with PCR, DMFT, number of decayed teeth, and total number of approximal caries.

Table 3 Correlation of oral conditions and salivary buffering capacity

\begin{tabular}{|c|c|c|c|c|c|c|c|c|c|c|c|}
\hline \multirow{2}{*}{$\begin{array}{c}\text { Salivary buffering } \\
\text { capacity }\end{array}$} & \multicolumn{2}{|c|}{$0(n=12)$} & \multicolumn{2}{|c|}{$1(n=70)$} & \multicolumn{2}{|c|}{$2(n=50)$} & \multicolumn{2}{|c|}{$3(n=25)$} & \multicolumn{3}{|c|}{ Total $(n=157)$} \\
\hline & mean & SD & mean & SD & mean & SD & mean & SD & mean & SD & $P$-value \\
\hline Age at the first visit & 37.830 & 13.849 & 38.640 & 13.349 & 35.280 & 11.137 & 33.680 & 11.817 & 36.720 & 12.518 & 0.300 \\
\hline $\mathrm{D}$ & 2.800 & 2.573 & 3.670 & 3.291 & 4.780 & 3.699 & 4.500 & 3.514 & 4.110 & 3.450 & 0.213 \\
\hline $\mathrm{M}$ & 0.800 & 1.033 & 1.790 & 2.603 & 1.540 & 1.982 & 2.000 & 2.654 & 1.680 & 2.343 & 0.853 \\
\hline $\mathrm{F}$ & 12.200 & 7.146 & 10.520 & 5.814 & 10.720 & 5.771 & 10.290 & 5.465 & 10.660 & 5.795 & 0.807 \\
\hline Total of flat surface caries & 0.580 & 1.505 & 1.810 & 3.906 & 3.180 & 4.797 & 3.640 & 5.155 & 2.450 & 4.367 & 0.004 \\
\hline Total of approximal caries & 2.750 & 2.958 & 4.640 & 6.249 & 7.900 & 7.833 & 6.200 & 6.212 & 5.780 & 6.771 & 0.026 \\
\hline PCR & 42.060 & 18.596 & 41.340 & 21.442 & 36.000 & 19.599 & 35.150 & 13.566 & 38.710 & 19.623 & 0.434 \\
\hline
\end{tabular}

Scores were evaluated for salivary buffering capacity using the chart of Dentobuff.

Score 0: immediately blue, Score 1: blue, Score 2: green, Score 3: orange

There were no statistically significant correlations for primary and secondary caries. The salivary buffering capacity showed a

significant correlation with the number of flat surface caries and approximal caries. 
To investigate the correlation of each dental caries condition and salivary levels of these bacteria, dental caries were divided into subgroups: primary caries and secondary caries; flat surface caries and approximal caries.

Table 4 Logistic regression analyses of MS

\begin{tabular}{lccc}
\hline \multicolumn{1}{c}{ MS } & Odds ratio & $\mathbf{9 5 \%}$ CI & $\boldsymbol{P}$-value \\
\hline Primary or Secondary & & & \\
Primary caries & 1.100 & $0.94-1.30$ & 0.230 \\
Secondary caries & 1.130 & $0.81-1.58$ & 0.460 \\
\cline { 2 - 4 } & & & \\
Flat or Approximal & & & \\
Flat surface caries & 1.210 & $0.77-1.92$ & 0.410 \\
Approximal caries & 1.090 & $0.76-1.55$ & 0.650 \\
\cline { 2 - 4 } Flat surface caries & & & \\
CA & & & \\
CB & 1.520 & $0.84-2.74$ & 0.170 \\
& 0.900 & $0.42-1.91$ & 0.780 \\
Approximal caries & & & \\
CA & & & \\
CB & 1.420 & $0.73-2.73$ & 0.300 \\
& 1.180 & $0.62-2.25$ & 0.620 \\
Multivariate Analyses & & & \\
Flat CA & & & \\
Flat CB & 1.390 & $0.71-2.70$ & 0.330 \\
Approximal CA & 0.650 & $0.21-1.99$ & 0.450 \\
Approximal CB & 1.180 & $0.57-2.48$ & 0.650 \\
\hline & & $0.56-3.95$ & 0.420 \\
\hline
\end{tabular}

CA: from the demineralization of enamel surface to dentinal caries CB: dental caries extending into the dental pulp and tooth stump There were no significant correlations for primary and secondary caries vs. salivary levels of MS

Table 5 Logistic regression analyses of LB

\begin{tabular}{lrrr}
\hline \multicolumn{1}{c}{ LB } & Odds ratio & $\mathbf{9 5 \%}$ CI & P-value \\
\hline Primary or Secondary & & & \\
Primary caries & 1.070 & $0.91-1.25$ & 0.410 \\
Secondary caries & 1.300 & $0.90-1.84$ & 0.170 \\
\cline { 2 - 4 } & & & \\
Flat or Approximal & & & \\
Flat surface caries & 2.100 & $1.29-3.41$ & 0.000 \\
Approximal caries & 0.930 & $0.64-1.34$ & 0.690 \\
\cline { 2 - 4 } & & & \\
Flat surface caries & & & \\
CA & 2.010 & $1.09-3.70$ & 0.030 \\
CB & 2.760 & $1.17-6.55$ & 0.020 \\
\cline { 2 - 4 } Approximal caries & & & \\
CA & & & \\
CB & 1.490 & $0.75-2.93$ & 0.250 \\
& 3.150 & $1.54-6.42$ & 0.000 \\
Multivariate Analyses & & & \\
Flat CA & & & 0.070 \\
Flat CB & 1.870 & $0.93-3.74$ & 0.080 \\
Approximal CA & 1.250 & $0.36-4.31$ & 0.720 \\
Approximal CB & 1.090 & $0.50-2.35$ & 0.830 \\
\hline
\end{tabular}

CA: from the demineralization of enamel surface to dentinal caries CB: dental caries extending into the dental pulp and tooth stump There were no statistically significant correlations found for primary and secondary caries.
Some teeth had both flat surface caries and approximal caries. To obviate the problem of confounding, multivariate logistic regression analysis was carried out. As shown in Tables 4, 5 and 6 , there were no statistically significant correlations for salivary MS level using Dentocult MS, salivary LB level using Dentocult LB and buffering capacity with primary and secondary caries. Statistically significant correlations were observed between salivary LB level using Dentocult LB and the number of flat surface caries $(P<0.001)$. Furthermore, to confirm dose response relations, flat surface caries and approximal caries were divided based on their progression (CA or $\mathrm{CB}$ ). Logistic regression analysis was again carried out. For salivary LB level using Dentocult LB and approximal caries, CB had a statistically significant correlation $(P<0.001)$. However, in the CA groups the correlation was not statistically significant. For salivary buffering capacity and flat surface caries, CA had a statistically significant correlation $(P<$ 0.030). In contrast, for MS, there were no statistically significant correlations in any group.

\section{Discussion}

The primary role of the salivary MS level, determined using Dentocult MS, salivary LB level using Dentocult LB and buffering capacity, is to predict the future incidence of dental caries. Many epidemiological studies have shown the utility of these tests (18-20,31). MS and LB inhabitant

Table 6 Logistic regression analyses of salivary buffering capacity

\begin{tabular}{lrcc}
\hline Salivary buffering capacity & Odds ratio & $\mathbf{9 5} \% \mathbf{C I}$ & $P$-value \\
\hline Primary or Secondary & & & \\
Primary caries & 1.149 & $0.90-1.35$ & 0.085 \\
Secondary caries & 0.980 & $0.71-1.33$ & 0.870 \\
\cline { 2 - 4 } Flat or Approximal & & & \\
Flat surface caries & & & \\
Approximal caries & 1.410 & $0.89-2.23$ & 0.140 \\
& 1.250 & $0.87-1.79$ & 0.230 \\
Flat surface caries & & & \\
CA & & & \\
CB & 1.930 & $1.07-3.48$ & 0.030 \\
& 1.730 & $0.80-3.73$ & 0.170 \\
Approximal caries & & & \\
CA & & & \\
CB & 1.370 & $0.70-2.65$ & 0.360 \\
& 1.850 & $0.97-3.54$ & 0.060 \\
Multivariate Analysis & & & \\
Flat CA & & & \\
Flat CB & 1.890 & $0.97-3.69$ & 0.060 \\
Approximal CA & 1.210 & $0.40-3.64$ & 0.740 \\
Approximal CB & 1.000 & $0.47-2.12$ & 1.000 \\
\hline
\end{tabular}

CA: from the demineralization of enamel surface to dentinal caries CB: dental caries extending into the dental pulp and tooth stump There were no statistically significant correlations for primary and secondary caries vs. salivary buffering capacity. 
caries lesions and are released in saliva, thus we evaluated the salivary MS level using Dentocult MS, salivary LB level using Dentocult LB and buffering capacity as clinical indicators of the present caries condition.

MS play an important role in the development and progression of dental caries (32). LB also plays a role in progression of dental caries (32). These bacteria are found in carious teeth. Previous studies have suggested that MS inhabit both deep regions of decayed teeth and the surface of teeth (33). In contrast, LB colonizes various parts of the oral cavity such as the oral mucosa, tongue dorsum, saliva and tooth surfaces (34). LB has been detected in high numbers in both superficial and deep caries (35). It was reported that many species of lactobacilli have been detected in carious dentine (36). Although these tests have been mostly evaluated in children, in this study we used them to study adults. A previous study reported that factors such as plaque scores, higher counts of MS, and lower buffering capacity contributed significantly to the higher risk profiles for adults compared to children (25). Based on the results of these studies, we evaluated MS, LB and buffering capacity as clinical indicators of the present dental caries condition in adult patients.

As the results of this study indicate, salivary MS level determined using Dentocult MS had no significant correlation with the number of approximal caries or flat surface caries. This may be because this bacterium is not always released into saliva on chewing and salivary levels of MS may not always represent actual tooth conditions. The salivary buffering capacity had no significant correlation with the number of caries (Tables 3 and 6). It is conceivable that salivary buffering capacity depends on the acidogenicity of bacteria including MS. On the other hand, salivary LB level determined using Dentocult LB did correlate significantly with the number of both flat and approximal caries. Some studies reported that the salivary level of LB is an important factor that predicts the incidence of root caries in elderly populations $(37,38)$. In our study, the ages of the subjects ranged from 20 to 63 . There are also reports that the prevalence and incidence of root caries in elderly people are higher than in younger people $(39,40)$. For adults, it is necessary to evaluate caries differently from children. For example, in adults one needs to consider the prosthetic restorations and periodontal status. In the present study, we substantiated the finding that LB detected in various parts of the oral cavity has a bearing on caries.

In conclusion, we found a correlation between salivary LB level determined using Dentocult LB and flat surface caries for several stages of caries. LB is not required for the development of lesions. Nonetheless, they may potently contribute to the demineralization of teeth once lesions are established on teeth. Salivary levels of LB may correspond to the stage of caries. In addition, clinically, secondary caries and approximal caries often remained unnoticed. Determination of the salivary LB level using Dentocult LB could be a useful tool to aid the detection of approximal and secondary caries.

\section{References}

1. Edwardsson S, Koch G, Obrink M (1972) Strep. sanguis, Strep. mutans and Strep. salivarius in saliva. Prevalence and relation to caries increment and prophylactic measures. Odontol Revy 23, 279-296

2. Jordan HV (1976) Cariogenic flora: establishment, localization, and transmission. J Dent Res $55 \mathrm{Spec}$ No: C10-14

3. McCormack-Brown KR, McDermott RJ (1991) Dental caries: selected factors of children at risk. Dent Assist 60, 10-14

4. Beighton D, Adamson A, Rugg-Gunn A (1996) Associations between dietary intake, dental caries experience and salivary bacterial levels in 12-yearold English schoolchildren. Arch Oral Biol 41, 271280

5. Hanada N (2000) Current understanding of the cause of dental caries. Jpn J Infect Dis 53, 1-5

6. Bender GR, Sutton SVW, Marquis RE (1986) Acid tolerance, proton permeabilities, and membrane ATPases of oral streptococci. Infect Immun 53, 331-338

7. Loesche WJ (1986) Role of the Streptococcus mutans in human dental decay. Microbiol Rev 50, 353-380

8. Van Houte J, Gibbons RJ, Pulkkinen AJ (1972) Ecology of human oral lactobacilli. Infect Immun 6, 723-729

9. Staat RH, Gawronski TH, Cressey DE, Harris RS, Folke LE (1975) Effects of dietary sucrose levels on the quantity and microbial composition of human dental plaque. J Dent Res 54, 872-880

10. Holbrook WP, de Soet JJ, de Graaff J (1993) Prediction of dental caries in pre-school children. Caries Res 27, 424-430

11. Crossner CG, Claesson R, Johansson T (1989) Presence of mutans streptococci and various types of lactobacilli in interdental spaces related to development of proximal carious lesions. Scand J Dent Res 97, 307-315

12. Loesche WJ, Syed SA (1973) The predominant cultivable flora of carious plaque and carious dentine. Caries Res 7, 201-216

13. Fitzgerald RJ, Adams BO, Fitzgerald DB, Knox 
KW (1981) Cariogenicity of human plaque lactobacilli in gnotobiotic rats. J Dent Res 60, 919926

14. Arneberg P, Ogaard B, Scheie AA, Rolla G (1984) Selection of Streptococcus mutans and lactbacilli in an intra-oral human caries model. J Dent Res 63, 1197-1200

15. Marsh PD, Featherstone A, McKee AS, Hallsworth AS, Robinson C, Weatherell JA, Newman HN, Pitter AF (1989) A microbiological study of early caries of approximal surfaces in schoolchildren. J Dent Res $68,1151-1154$

16. Jensen B, Bratthall D (1989) A new method for the estimation of mutans streptococci in human saliva. J Dent Res. 68, 468-471

17. Pienihakkinen K, Jokela J (1995) A simple method for monitoring mutans streptococci in young children. Eur J Oral Sci 103, 61-62

18. Oncag O, Alpoz AR, Eronat C (2000) Salivary Streptococcus mutans, Lactobacilli levels and buffer capacity in children with esophageal burns. J Clin Pediatr Dent 24, 147-151

19. Takano N, Ando Y, Yoshihara A, Miyazaki H (2003) Factors associated with root caries incidence in an elderly population. Community Dent Health 20, 217-222

20. Shi S, Deng Q, Hayashi Y, Yakushiji M, Machida Y, Liang Q (2003) A follow-up study on three caries activity tests. J Clin Pediatr Dent 27, 359-364

21. Holbrook WP (1993) Dental caries and cariogenic factors in pre-school urban Icelandic children. Caries Res 27, 431-437

22. Loesche WJ, Eklund S, Earnest R, Burt B (1984) Longitudinal investigation of bacteriology of human fissure decay: epidemiological studies in molar shortly after eruption. Infect Immun 46, 765-772

23. Tagliaferro EP, Pereira AC, Meneghim Mde C, Ambrosano GM (2006) Assessment of dental caries predictors in a seven-year longitudinal stduy. $\mathrm{J}$ Public Health Dent 66, 169-173

24. Batoni G, Ota F, Ghelardi E, Senesi S, Barnini S, Freer G, Hirota K, Gabriele M, Marcucci M, Campa M (1992) Epidemiological survey of Streptococcus mutans in a group of adult patients living in Pisa (Italy). Eur J Epidemiol 8, 238-242

25. Petersson GH, Fure S, Twetman S, Bratthall D (2004) Comparing caries risk factors and risk profiles between children and elderly. Swed Dent J 28, 119128

26. Narhi TO, Kurki N, Ainamo A (1999) Saliva, salivary micro-organisms, and oral health in the home-dwelling old elderly - a five-year longitudinal study. J Dent Res 78, 1640-1646

27. Guivante-Nabet C, Berenholc C, Berdal A (1999) Caries activity and associated risk factors in elderly hospitalised population - 15-months follow-up in French institutions, Gerodontology 16, 47-58

28. Barmes DE, Infirri JS (1977) WHO activities in oral epidemiology. Community Dent Oral Epidemiol 5, 22-29

29. Reis IM, Flack VF, Atchison KA, White SC (1998) Findings of clinical and radiographic caries among several adult age groups. Oral Surg Oral Med Oral Pathol Oral Radiol Endod 86, 760-764

30. Poorterman JH, Aartman IH, Kieft JA, Kalsbeek H (2000) Value of bite-wing radiographs in a clinical epidemiological study and their effect on the DMFS index. Caries Res 34, 159-163

31. Splieth C, Bernhardt O (1999) Prediction of caries development for molar fissures with semiquantitative mutans streptococci test. Eur J Oral Sci 107, 164169

32. Nomura Y, Tsuge S, Hayashi M, Sasaki M, Yamaguchi T, Ueda N, Hanada N (2004) A survey on the risk factors for the prevalence of dental caries among preschool children in Japan. Pediatr Dent J $14,79-85$

33. Babaahmady KG, Challacombe SJ, Marsh PD, Newman HN (1998) Ecological study of Streptococcus mutans, Streptococcus sobrinus and Lactobacillus spp. at sub-sites from approximal dental plaque from children. Caries Res 32, 51-58

34. Takei M, Kobayashi Y, Iwasaki S, Fujihashi T (1971) Distribution of lactobacilli in oral cavities. Jpn J Microbiol 15, 109-112

35. Hahn CL, Falkler WA Jr, Minah GE (1991) Microbiolobgical studies of carious dentine from human teeth with irreversible pulpitis. Arch Oral Biol $36,147-153$

36. Byun R, Nadkarni MA, Chhour KL, Martin FE, Jacques NA, Hunter N (2004) Quantitative analysis of diverse Lactobacillus species present in advanced dental caries. J Clin Micobiol 42, 3128-3136

37. Powell LV, Leroux BG, Persson RE, Kiyak HA (1998) Factors associates with caries incidence in an elderly population. Community Dent Oral Epidemiol 26, 170-176

38. Scheinin A, Pienihakkinen K, Tiekso J, Holmberg S, Fukuda M, Suzuki A (1994) Multifactorial modeling for root caries prediction: 3-year followup results. Community Dent Oral Epidemiol 22, 126129 
39. Fure S (1997) Five-years incidence or coronal and root caries in 60-, 70- and 80-year-old Swedish individuals. Caries Res 31, 249-258
40. Locker D, Leake JL (1993) Coronal and root decay experience in older adults in Ontario, Canada. J Public Health Dent 53, 158-164 